\title{
A NOTE ON FINITE SOLVABLE $K$-GROUPS
}

\author{
HOMER BECHTELL
}

A finite $K$-group is a group having a complemented subgroup lattice i.e. if $A$ is a subgroup of a finite $K$-group $G$, there exists a subgroup $B$ such that $G=\{A, B\}, A \cap B=1$. The purpose of this note is to identify the structure of a solvable $K$-group. This reduces to an investigation of a primitive linear solvable $K$-group which is identified as a semidirect product of a cyclic group of square-free order by a cyclic group of square-free order.

Only finite groups will be considered. The notation and the terminology will be that found in the standard references with clarification made whenever necessary.

Nilpotent $K$-groups are a direct product of elementary Abelian $p$ groups and the supersolvable $K$-groups are a subgroup of a direct product of groups of square-free order (e.g. see Suzuki [5]). Coupling these results with one of Gaschütz [1], a group splits over each normal subgroup whenever all the Sylow $p$-subgroups are elementary Abelian, and another by $\mathrm{F}$. Gross [3], a solvable group is a $K$-group if and only if the group splits over each normal subgroup, a defining property for a class of solvable $K$-groups based on the structure of the Sylow $p$-subgroups is obtained. However this class does not exhaust the set of solvable $K$-groups as the symmetric group of degree four indicates. So the remaining portion of this note will complete the classification.

Zacher [6] has shown the following:

(1) A finite solvable group $G$ is a $K$-group, if and only if $G$ contains a series of normal subgroups $1=N_{0}<N_{1}<\cdots<N_{r}=G$ such that each $N_{i+1} / N_{i}$ is a maximal normal nilpotent subgroup of $G / N_{i}$, and $\Phi\left(G / N_{i}\right)=1$ for $i=0,1, \cdots, r-1$.

(2) Each homomorphic image of a solvable $K$-group is a $K$-group. From these the following results of F. Gross [3] can be developed:

(3) A solvable group is a $K$-group if and only if the group splits over each normal subgroup.

(4) Each normal subgroup of a $K$-group is a $K$-group.

Note that for (3), if $G$ is a $K$-group then it splits over each normal subgroup. On the other hand it is enough to note that if $G$ splits over each normal subgroup then $\Phi(G)=1$. Moreover since $G$ is solvable, then a series of subgroups $1=N_{0}<N_{1}<\cdots<N_{r}=G$ exists such that

Received by the editors March 30, 1966. 
$N_{i+1} / N_{i}$ is the maximal nilpotent normal subgroup of $G / N_{i}$. Since for each $i$, there exists a subgroup $M_{i}$ such that $G=N_{i} M_{i}, N_{i} \cap M_{i}=1$, then $M_{i}$ also splits over each normal subgroup. Consequently $\Phi\left(G / N_{i}\right)$ is the identity and from (2) the result follows that $G$ is a $K$-group.

As for (4) note that by a known result of Gaschütz [2] if a group has the Frattini subgroup the identity element then so does each normal subgroup. So if $G$ is a solvable $K$-group and $N$ is a normal subgroup of $G$, then in $N$ a series satisfying (2) exists. Thus $N$ is a $K$-group.

In general, a solvable group $G$ is a subdirect product of solvable groups $H$ such that $H$ contains precisely one minimal normal subgroup. However if also $G$ is a $K$-group and $A$ is the kernel of the projection of $G$ on to $H$, there exists a subgroup $B$ of $G$ such that $G=A B$, $A \cap B=1$, and $B \cong H$ i.e. the direct factors in the subdirect product are isomorphic to subgroups of $G$. By (2), $H$ is a $K$-group. Furthermore,

THEOREM 1. A group $G$ is a solvable $K$-group if and only if $G$ is a subdirect product of a finite collection of $K$-groups $H$, such that each $H$ is isomorphic to a subgroup of $G$ and each $H$ possesses a unique minimal normal subgroup.

Proof. The necessity of the conditions follows from the preceding remarks.

Consider then a subdirect product $G \leqq H_{1} \otimes H_{2} \otimes \cdots \otimes H_{n}$, each $H_{i}$ being a solvable $K$-group possessing a unique minimal normal subgroup, for which the projections $\pi_{i}$ exist, $G \pi_{i}=H_{i}$, having kernels $A_{i}$, such that $\cap_{I} A_{i}=1$, for all $i \in I=\{1, \cdots, n\}$. Since $\Phi\left(H_{i}\right)=1$, and $\Phi(G) \pi_{i} \leqq \Phi\left(H_{i}\right)=1$ for all $i \in I$, then $\Phi(G) \leqq \bigcap_{I} A_{i}=1$. Therefore this and the solvability of $G$ implies that $G$ splits over each minimal normal subgroup. So denote by $N$ a normal subgroup of $G$ such that for each $G$-normal subgroup $N^{*}<N, G$ splits over $N^{*}$. Then there exists a subgroup $C^{*}$ of $G$ such that $N^{*} C^{*}=G, N^{*} \cap C^{*}$ $=1$. Moreover $N=N^{*}\left(N \cap C^{*}\right), N / N^{*}$ is a minimal normal subgroup of $G / N^{*}$, and thus $N \cap C^{*}$ is a minimal normal subgroup of $C^{*}$. Then note that $H_{i}=\left(N^{*} \pi_{i}\right)\left(C^{*} \pi_{i}\right),\left(N^{*} \pi_{i}\right) \cap\left(C^{*} \pi_{i}\right)=1$ for all $i \in I$. However $H_{i}$ a $K$-group implies that $C^{*} \pi_{i}$ is a $K$-group and hence $\Phi\left(C^{*} \pi_{i}\right)$ $=1$. Hence for each $i \in I, \Phi\left(C^{*}\right) \leqq A_{i}$. So $\Phi\left(C^{*}\right)=1$. From this follows the existence of a subgroup $B$ of $C^{*}$ such that $C^{*}=\left(N \cap C^{*}\right) B$, $\left(N \cap C^{*}\right) \cap B=1$ and also that $G=N^{*}\left(N \cap C^{*}\right) B=N B, N \cap B=1$. Thus $G$ splits over each normal subgroup. With this and (3) above, $G$ is a $K$-group. 
In order to examine solvable $K$-groups $G$ having a unique minimal normal subgroup $N$, it should first be noted that the maximal nilpotent normal subgroup of $G$, the Fitting subgroup $F(G)$, is $N$. This follows from $F(G)$ being a direct product of minimal normal subgroups of the $K$-group $G$. Furthermore since in a solvable group the centralizer of $F(G)$ is contained in $F(G)$, then $F(G)$ is its own centralizer. Consequently for some prime $p,|F(G)|=p^{n}$, and there exists a subgroup $H$ such that $G=F(G) H, F(G) \cap H=1$, and $H$ can be considered as an irreducible linear group over a field of $p$ elements acting faithfully on $F(G)$. So let us examine $H$ as an irreducible linear group.

Denote the full linear group on an $n$-dimensional vector space $V^{n}$ over a field of $p$ elements by $G L(n, p)$. An irreducible subgroup $G$ of $G L(n, p)$ is imprimitive if $V^{n}$ can be expressed as a direct sum $V_{1} \oplus \cdots \oplus V_{k}, k>1$, of subspaces $V_{j}$, that are permuted by the elements of $G$ : otherwise $G$ is primitive on $V^{n}$. The systems of imprimitivity, the $V_{\boldsymbol{j}}$, are permuted transitively since $G$ is irreducible. Furthermore by choosing the system of imprimitivity to be a minimum, the subgroup $G_{i}$ of $G$ that leaves invariant $V_{i}$ induces on $V_{i}$ an irreducible primitive subgroup $G_{i}^{*}$ of $G L(n / k, p) . G$ is then contained in the group $A=B C, B \cap C=1, B$ normal in $A, B$ isomorphic to a direct product of $k$ copies of a primitive solvable subgroup of $G L(n / k, p)$, and $C$ isomorphic to a certain solvable transitive subgroup of the symmetric group of degree $k$. Also each primitive solvable subgroup $S$ of $G L(n, p)$ is contained in a maximal solvable subgroup of $G L(n, p)$ and each subgroup of $G L(n, p)$ containing $S$ is primitive.

Theorem 2. A primitive solvable linear group of $G L(n, p)$ is a $K$ group if and only if it is the semidirect product of a cyclic group of square-free order by a cyclic group of square-free order.

Proof. A semidirect product $H$ of a cyclic group of square-free order by a cyclic group of square-free order has elementary Abelian Sylow $p$-subgroups and hence, by a result of Gaschütz [1], splits over each normal subgroup. By (3) above this is sufficient for $G$ to be a $K$-group.

On the other hand note that $H$ a solvable $K$-group implies that $F(H)$ is elementary Abelian and the maximal normal Abelian subgroup of $H$. Then it follows that $F(H)$ is a subgroup of the multiplicative group of a finite field (see [4]). So $F(H)$ is cyclic and $\Phi(F(H))=1$ implies that $F(H)$ is square-free. It is also known (see [4]) that whenever $G$ is a maximal primitive subgroup of $G L(n, p)$ and $A$ is the maximal normal Abelian subgroup of $G$ that $G / A$ is cyclic. Consequently if $H$ is a primitive subgroup, $H \leqq G$, then $A H / A \cong H / A \cap H$ 
implies that $H$ is a semidirect product of a cyclic group of square-free order be a cyclic group. By (2), $|H / A \cap H|$ is square-free.

Corollary 2.1. A $K$-group $G$ that is a solvable irreducible subgroup of $G L(n, p)$ is a semidirect product of a subgroup $A$ by a subgroup $B$ such that

(i) $A$ is the direct product of $k$ copies of a group $H$ that is the semidirect product of a cyclic group of square-free order by a cyclic group of square-free order and

(ii) $B$ is a K-group isomorphic to a solvable transitive subgroup of the symmetric group of degree $k$.

Proof. The semidirect product exists from the earlier remarks and the application of the above theorem to $G$ suffices for the result. The transitive subgroup is determined by the systems of imprimitivity on $V^{n}$ relative to $G$.

An alternative representation of a solvable $K$-group $G$ having a unique minimal normal subgroup $N$ is that $G$ can be considered as a primitive permutation group having $\operatorname{deg}(G)=|N|=p^{n}$ for some prime $p$. The results of the previous theorem and corollary can then be applied using the relationship between irreducible subgroups and primitive permutation groups.

I thank Fritz Gross for letting his results be known to me.

\section{BIBLIOGRAPHY}

1. W. Gaschütz, Zur Erzeiterungstheorie der endlichen Gruppen, J. Reine Angew. Math. 190 (1952), 93-107.

2. 170.

3. F. Gross, Überaufiösbare Gruppen mit elementar-abelschen Sylowgruppen, Ph.D. Thesis, Christian-Albrechts-Universität, Kiel, 1962.

4. D. Suprunenko, Soluble and nilpotent linear groups, Transl. Math. Monos., Vol. 9, Amer. Math. Soc., Providence, R. I., 1963.

5. M. Suzuki, Structure of a group and the structure of its lattice of subgroups, Springer, Berlin, 1956.

6. G. Zacher, Caratterizzazione dei gruppi risolubili d'ordine finito complementarı, Rend. Sem. Mat. Univ. Padova 22 (1953), 113-122.

University of New Hampshire 